\section{Human biomarkers: can they help us to develop a new tuberculosis vaccine?}

\author{
Helen A Fletcher ${ }^{*, 1} \&$ Hazel M Dockrell**,1
}

The most effective intervention for the control of infectious disease is vaccination. The BCG vaccine, the only licensed vaccine for the prevention of tuberculosis (TB) disease, is only partially effective and a new vaccine is urgently needed. Biomarkers can aid the development of new TB vaccines through discovery of immune mechanisms, early assessment of vaccine immunogenicity or vaccine take and identification of those at greatest risk of disease progression for recruitment into smaller, targeted efficacy trials. The ultimate goal, however, remains a biomarker of TB vaccine efficacy that can be used as a surrogate for a TB disease end point and there remains an urgent need for further research in this area.

First draft submitted: 13 May 2015; Accepted for publication: 11 March 2016;

Published online: 20 May 2016

It is clear that new tools are needed to achieve the ambitious goals of controlling tuberculosis (TB) set for 2025 and 2035 by the World Health Assembly, with the vision of a world free of TB [1,2]. It is estimated that, with optimized use of current tools, universal health coverage and improved social protection, we can achieve a rate of $25 \mathrm{~TB}$ cases per 100,000 a year by 2035 but, to 'end TB' by 2035, we will need new diagnostics, drug treatments and vaccines. It is also clear that biomarkers can play a role in the development of the new tools needed to end TB. This review will discuss what types of biomarkers and biosignatures are needed, and report on recent progress and new developments.

To start, it may be useful to define biomarkers. Biomarkers can be indicators of disease or of host immunity. In many or most cases, a combination of biomarkers will be needed rather than measurement of a single analyte, comprising a biosignature. In some cases, such biosignatures can act as surrogate markers for the protective efficacy of vaccines, as correlates of protection. In TB, these biomarkers can be developed to help control in different ways, some of which have made considerable progress in the last few years.

\section{Biomarkers of vaccine efficacy}

To control TB, a new and more protective vaccine than BCG is a priority. Although the first efficacy trial of the first new subunit candidate vaccine for the prevention of disease, MVA85A, has not shown significant protective efficacy when given to BCG-vaccinated infants in South Africa [3]; there is a pipeline of other new vaccine candidates [4]. If we could predict the likely efficacy of these vaccine candidates it would help to select the most promising candidates in preclinical or early clinical trials, reducing the risks associated with vaccine failure. To improve vaccine candidate selection we could improve animal models, use human challenge models and identify biomarkers

'London School of Hygiene \& Tropical Medicine, London, W1CE 7HT, UK

*Author for correspondence: Tel.: +44 0207927 2666; helen.fletcher@lshtm.ac.uk

**Author for correspondence: Tel.: +44 0207927 2466; hazel.dockrell@lshtm.ac.uk

\section{KEYWORDS}

- BCG • biomarker correlate

- tuberculosis $\bullet$ vaccine 
that correlate with $\mathrm{TB}$ vaccine efficacy. To date, correlates of protection have not been identified, although further results from studies in both BCG-vaccinated infants and adolescents and MVA85A-boosted infants are awaited. In a study by Kagina et al., measurement of polyfunctional T cells in BCG-stimulated whole blood cultures from BCG-vaccinated infants at 10 weeks of age did not discriminate between infants who subsequently progressed to $\mathrm{TB}$ disease and those who remained healthy, using intracellular cytokine staining and flow cytometry [5]. Polyfunctional $\mathrm{T}$ cells may not be protective, but it is also possible that in the Kagina study these cells were measured at the wrong time point, or the sample size may have been too small to see an effect. In a more recent study, BCG-specific IFN- $\gamma$ measured by ELIspot was found to be associated with reduced risk of developing TB disease in the same South African infant population [6]. Although we have no confirmed or refuted correlate due to the lack of efficacy with MVA85A, there is now low confidence in all TH1-boosting $\mathrm{TB}$ vaccine candidates and this has had a significant impact on the TB vaccine pipeline (Figure 1). In 2013, there were 13 vaccine candidates in active clinical development for TB disease prevention. Although there are currently ongoing $\mathrm{TB}$ vaccine clinical trials (ClinicalTrials.gov), many of these trials are research studies only, for example, investigation of new routes of vaccine delivery or for development of a human challenge model and the vaccine candidates used are no longer considered on a pathway toward efficacy testing. There has only been one new entry into the TB Vaccine pipeline since 2013 (DAR 901) resulting in an overall contraction of the pipeline by $40 \%$ from 2013 to 2015 . This is a concern as given that we have no confirmed biomarkers of vaccine efficacy; we need more data and should, therefore, be performing more clinical vaccine trials and not less.

\section{Biomarkers of vaccine immunogenicity}

Even though measuring cytokines has not to date provided a correlate of protection, these assays are very useful as biomarkers of vaccine immunogenicity. IFN $-\gamma$ measured in diluted whole blood assays stimulated with purified protein derivative (PPD), provides a clear indication of BCG vaccination immunogenicity or 'take' in UK infants [8], while the IFN- $\gamma$ ELISPOT was used in the early trials developing the MVA85A vaccine [9]. These assays can aid in dose finding and identifying the timing of any peak of immune responses following vaccination, thus contributing to decisions about the best time to administer a boosting vaccine. Thus we already have biomarkers that can help in the development of those new TB vaccine candidates in the development pipeline. However, these assays cannot, as so far performed, provide a correlate of protection, as they have neither been proved nor disproved as associating with vaccine induced protection.

\section{Biomarkers for prevention of infection}

The lack of TB vaccine efficacy trials is a direct consequence of the cost of performing such trials. Following exposure and infection with Mycobacterium tuberculosis there is a $10 \%$ lifetime risk of developing disease, although the greatest risk is within the first 2 years following exposure [10]. In endemic populations, the annual incidence of disease is typically $1-2 \%$ requiring large numbers and lengthy follow-up to obtain sufficient sample size for an immune correlates analysis with a TB disease end point. One alternative is to use infection as an end point for immune correlates studies (prevention of infection [POI]) [11]. The annual incidence of TB infection is approximately five to tenfold higher than that of TB disease, substantially reducing the size and length of a vaccine efficacy trial [11]. Although attractive, there are caveats to this approach. We have limited tools available for the identification of TB infection (currently only the TST and IGRA) [12]. In addition, prevention of infection may not correlate with prevention of disease and there is a risk that candidates which could prevent disease (but not infection) will be missed. Vaccine candidates that show promise in a POI trial will have to be evaluated in a trial with a TB disease end point to confirm effectiveness against active disease [12].

\section{Biomarkers for BCG replacement vaccines}

Although we need a more effective vaccine for protection against $\mathrm{TB}$, it is important to remember that BCG does provide protection against $\mathrm{TB}$ in some age groups and some populations [13]. In a recent meta-analysis of the literature, Mangtani et al. have shown that BCG protects in infancy and in those with low exposure to mycobacteria (measured by skin test reaction to PPD). The protective effect of BCG decreases with age of immunization and distance from the equator, likely because exposure to 


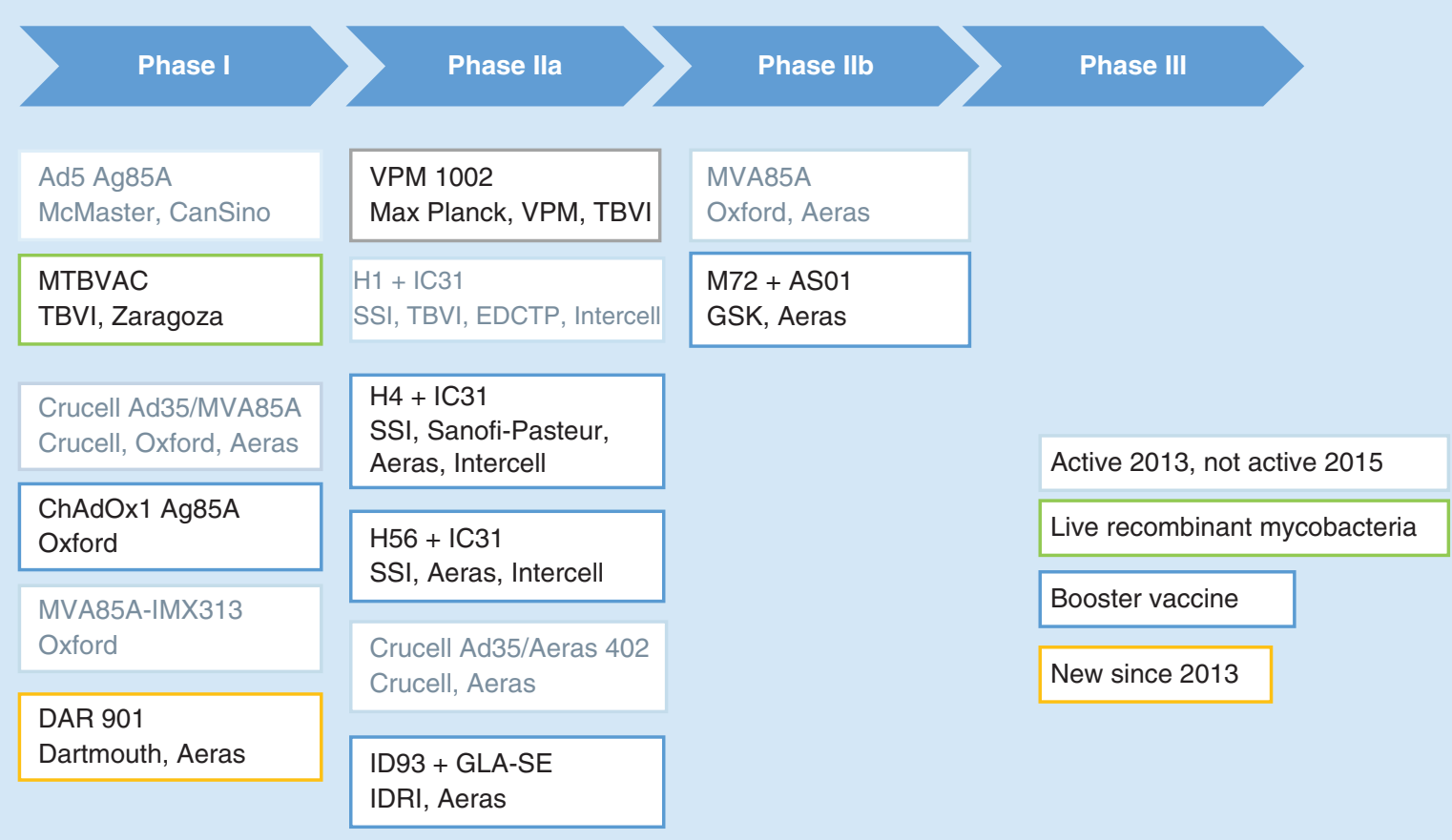

Figure 1. The global pipeline of tuberculosis vaccine candidates 2013-2015. Vaccines for prevention of tuberculosis disease or prevention of infection in active clinical development 2013-2015. This does not include therapeutic vaccines.

Data taken from [7].

environmental mycobacteria or $M$. tuberculosis increases as age and proximity to the equator increase. In populations where BCG is protective the effect can last for up to 20 years or even longer [14]. Therefore, if we are to replace BCG with an alternative live attenuated vaccine we must ensure that the protective efficacy of the replacement vaccine is at least the same (noninferior) as that of BCG. The identification of a biomarker of BCG efficacy, which could then be measured in clinical trials of replacement vaccines, would greatly aid the development of a BCG replacement vaccine. In addition, there is evidence that BCG can provide nonspecific protection against all-cause mortality in infant populations in West Africa [15-19]. The exact mechanism of this protective effect is unknown although recent literature suggests that BCG may drive epigenetic changes in innate immune cells, improving innate capacity to control infection with other pathogens [20-22]. Thus, if we are to replace BCG we may have to show that the replacement vaccine is both noninferior in ability to protect against TB and in ability to protect against all-cause mortality. Conducting such trials with clinical end points would be prohibitively lengthy and costly and there is an urgent need to identify biomarkers of the nonspecific effects of BCG, which can then be measured in clinical trials of BCG replacement vaccines.

\section{New approaches using mycobacterial growth inhibition}

Another recent approach goes back to the core of the matter, the ability to kill or inhibit the growth of mycobacteria. These assays measure the summative ability of all innate, cell-mediated and humoral immune components in a sample to contribute to the control of mycobacterial growth. The exact immune mechanism does not need to be identified and the assay can be transferred between different animal species and into humans, providing a bridge between preclinical efficacy of vaccine candidates and potential for efficacy in early clinical trials. Assays measuring mycobacterial growth have been used in a number of studies in recent decades, but have not proved easy to implement at scale or in vaccine trial settings [23-26]. However, mycobacterial growth can now be quantitated automatically using a mycobacterial growth inhibition assay, measuring the time to positivity using equipment available in many microbiology laboratories [27]. Although further optimization of 
this assay is needed, early indications are that BCG vaccination can induce the inhibition of mycobacterial growth [27]. Another approach is to perform this experiment in vivo in a human challenge study. This has been done by taking a biopsy of the BCG vaccination site and quantifying the BCG present [28-30]. Future studies will seek to challenge human volunteers with attenuated strains of $M$. tuberculosis either by the intradermal or pulmonary routes although significant safety and regulatory concerns will have to be addressed before this can be done.

\section{Biomarkers of infection versus disease}

Biomarkers of infection or of risk of progression to disease could also contribute to both the development of diagnostic assays for TB and help to identify those at greater risk of progression, thus enabling smaller and less costly vaccine trials. Advances in flow cytometry have given us the ability to measure multiple markers expressed on both the surface and inside immune cells. Using combinations of cell surface markers and intracellular cytokines or transcription factors, we are able to characterize the cellular immune response in TB infection and disease to identify biomarkers of disease stage. TB disease stage can be identified through flow cytometry detection of monofunctional (single cytokine-expressing) or polyfunctional (multiple cytokine-expressing) T cells. Polyfunctional $\mathrm{T}$ cells are increased in TB patients [31,32] and cells have been shown to be functionally and phenotypically different between TB patients and $M$. tuberculosis infected individuals [33-36]. However, whether flow cytometry can predict those at risk of developing future TB disease has not been tested.

\section{Biomarkers of infection \& treatment response: the power of gene expression signatures}

There has been considerable progress using gene signatures as biomarkers for identifying infection or monitoring treatment response. These include signatures for active TB identified in a number of studies [37-41]. Such signatures can work in both HIV positive and negative subjects, and in different geographical settings. Other ongoing studies aim to identify signatures in cohorts of either adolescents or adults in highly TB-endemic settings [42]. Interestingly, such signatures can discriminate between active disease and infection and, thus, may improve on the existing diagnostic tests, such as skin testing, the QuantiFERON TB Gold In-tube test (Cellestis, Australia) or T-SPOT ${ }^{\circledR}$.TB assay (Oxford Immunotec). A gene signature that would identify those progressing to clinical disease would enable early diagnosis and treatment, thus reducing transmission. Biosignatures that identify those at risk of developing disease before subclinical disease was present would, as noted above, help stratify participants in vaccine trials.

Gene signatures that normalize after successful treatment of clinical TB may also give clues about biomarkers of infection [37-38,43]. Some individuals relapse following apparently successful treatment, whereas most do not. Those with $\mathrm{TB}$ and diabetes mellitus are more difficult to cure and more likely to relapse than those with TB alone [44]. Such differences may provide additional indicators of what is needed to prevent relapse. In summary, there may be a number of complementary approaches that could help us identify the components of a protective immune response. Meanwhile technology is developing too, and it is likely that by the time the component parts of protective immunity have been identified, there will be simple and innovative ways in which to measure them in vaccine trials.

However, the 'Holy Grail' remains the identification of correlates of protection. It is sometimes said that we understand nothing about protection against $\mathrm{TB}$, but this is not the case. Mice lacking the ability to make or respond to IFN- $\gamma$ are more susceptible to disease as are humans with rare mutations in the IFN- $\gamma /$ IL-12/STAT1 signaling pathways [45-51]. We know that those with reduced CD4 T-cell numbers and function due to HIV infection become susceptible to TB. However, simple measures of CD4 T cells making IFN- $\gamma$, IL 2 and TNF- $\alpha$ have not so far proved to provide a protective biosignature $[3,52]$, although to date these have only been tested in one study as a correlate of TB risk [52].

\section{The way forward}

One issue here is that in the absence of clinical efficacy trials, how can such protective biosignatures be identified? BCG does provide efficacy in some populations including infants and skin test negative school children [13]. Studies of BCG in these populations of sufficient size and length to include a TB disease end point could provide the case-control samples required for biomarker identification. Analysis of BCG specific immune correlates in samples from the failed MVA85A 
infant efficacy trial provide an opportunity to identify biomarkers [3]. However, following the failure of the infant MVA85A trial, an on-going efficacy trial of MVA85A in HIV positive adults was downgraded to an extended safety study and there is now limited sample size for an immune correlates analysis in this study [53]. The only on-going efficacy trial with a TB disease end point is currently being performed with the vaccine candidate M72 in AS02 adjuvant. In this trial samples are being stored from all participants for retrospective biomarker analysis and it is critical that enough TB cases accrue during this trial to enable robust biomarker analysis in a case-control study. If this trial is terminated prematurely it could be decades before there is another opportunity to perform a correlates of risk study in a TB vaccine efficacy trial cohort.

\section{Conclusion}

Developing biomarkers of protective immunity is hard, when we do not have a full understanding of the host response in TB. At the present time, we seem closer to identifying biosignatures of infection and disease risk rather than of protection. Hopefully, there will be more to learn from ongoing studies, and particularly from both ongoing and future vaccine trials. We are obtaining a more objective view of the immunology of TB, guided by results from gene expression analysis using microarrays and now RNAseq. Measuring what really matters, the ability to kill or inhibit the growth of BCG or $M$. tuberculosis itself, may help, and also identify useful biomarkers. There are new approaches that should be explored, including the recent concepts of 'memory' or imprinting in innate immunity following BCG vaccination [20], more in depth analyses of memory $\mathrm{T}$ cells including epigenetic approaches, exploration of humoral immunity and insights from systems biology. To bring these different approaches together, some coordination of effort will also be useful, as can be achieved through biomarker work packages in large research consortia, such as the TBVAC2020 consortium recently funded by the EU [54], or the plans for a Global TB Vaccine Partnership to provide coordination between ongoing work funded by the Bill \& Melinda Gates Foundation and that funded by the EU.

\section{Future perspective}

In the next 5-10 years, there will be reinvigoration of the TB vaccine clinical trials pipeline with the entry of candidate $\mathrm{TB}$ vaccines funded through Horizon 2020, the Bill \& Melinda Gates Foundation, EDCTP and other funders. There is a drive to develop $\mathrm{TB}$ vaccine candidates that are not focused on boosting a Th1-type immune response, although the Th1 concept has been neither confirmed or refuted as important in vaccine induced protection. There is an urgent need for biomarkers that can select the most promising of these TB vaccine candidates for accelerated clinical development. A rapid and immediate acceleration in biomarker research is required in preparation for the screening of large numbers of new entry TB vaccine candidates in the next $5-10$ years.

\section{Financial \& competing interests disclosure}

The authors have no relevant affiliations or financial involvement with any organization or entity with a financial interest in or financial conflict with the subject matter or materials discussed in the manuscript. This includes employment, consultancies, honoraria, stock ownership or options, expert testimony, grants or patents received or pending, or royalties.

No writing assistance was utilized in the production of this manuscript.

\section{Open access}

This work is licensed under the Attribution-Non CommercialNoDerivatives 4.0 Unported License. To view a copy of this license, visit http://creativecommons.org/licenses/ by-nc-nd/4.0/

\section{EXECUTIVE SUMMARY}

- Biomarkers can aid the development of new tuberculosis (TB) vaccines in multiple ways.

- Biomarker research has identified immune mechanisms of TB disease that have potential importance for the design of new TB vaccine candidates.

- Monitoring of TB vaccine immunogenicity can be performed effectively with biomarkers.

- Biomarkers can be used to shorten the length of TB vaccine efficacy trials by focusing recruitment on individuals at greatest risk of developing TB disease.

- The 'Holy Grail' remains a biomarker of TB vaccine efficacy that can be used as a surrogate for a TB disease end point. 


\section{References}

Papers of special note have been highlighted as: •• of considerable interest

1 Lonnroth K, Migliori GB, Abubakar I et al. Towards tuberculosis elimination: an action framework for low-incidence countries. Eur. Respir. J. 45(4), 928-952 (2015).

2 WHO www.who.int/tb/post2015_strategy/en

3 Tameris MD, Hatherill M, Landry BS et al. Safety and efficacy of MVA85A, a new tuberculosis vaccine, in infants previously vaccinated with BCG: a randomised, placebo-controlled Phase $2 \mathrm{~b}$ trial. Lancet 381(9871), 1021-1028 (2013).

-• The first efficacy trial of a subunit tuberculosis $(\mathrm{TB})$ vaccine. This vaccine was designed to boost Th1 responses.

4 TuBerculosis Vaccine Initiative. www.tbvi.eu

5 Kagina BM, Abel B, Scriba TJ et al. Specific $\mathrm{T}$ cell frequency and cytokine expression profile do not correlate with protection against tuberculosis after Bacillus Calmette-Guerin vaccination of newborns. Am. J. Respir. Crit. Care Med. 182(8), 1073-1079 (2010).

- Study finding that Th1 cells were not associated with reduced risk of TB disease. This and the Tameris study have thrown doubt on the ability of vaccine-induced Th1 cells to protect.

6 FletcherHA, Snowden MA, Landry B et al. T-cell activation is an immune correlate of riskin BCG vaccinated infants. Nat. Commun. 12, 11290 (2016)

7 ClinicalTrials.gov. https://clinicaltrials.gov

8 Lalor MK, Ben-Smith A, Gorak-Stolinska P et al. Population differences in immune responses to Bacille Calmette-Guerin vaccination in infancy. J. Infect. Dis. 199(6), 795-800 (2009).

9 Mcshane H, Pathan AA, Sander CR et al. Recombinant modified vaccinia virus Ankara expressing antigen 85A boosts BCG-primed and naturally acquired antimycobacterial immunity in humans. Nat. Med. 10(11), 1240-1244 (2004).

10 Ferebee SH. Controlled chemoprophylaxis trials in tuberculosis: a general review. Bibl. Tuberc. 26, 28-106 (1970).

11 Ellis RD, Hatherill M, Tait D et al. Innovative clinical trial designs to rationalize $\mathrm{TB}$ vaccine development. Tuberculosis (Edinb.) 95(3), 352-357 (2015).

12 Vaccine Prevention of Sustained Mycobacterium Tuberculosis Infection
Summary Group. Developing vaccines to prevent sustained infection with Mycobacterium tuberculosis: conference proceedings: November 7, 2014, National Institute of Allergy and Infectious Diseases, Rockville, Maryland, USA. Vaccine 33(26), 3056-3064 (2015).

13 Mangtani P, Abubakar I, Ariti C et al. Protection by BCG vaccine against tuberculosis: a systematic review of randomized controlled trials. Clin. Infect. Dis. 58(4), 470-480 (2014).

14 Nguipdop-Djomo P, Heldal E, Rodrigues LC, Abubakar I, Mangtani P. Duration of BCG protection against tuberculosis and change in effectiveness with time since vaccination in Norway: a retrospective population-based cohort study. Lancet Infect. Dis. 16(2), 219-226 (2015).

15 Aaby P, Benn CS, Nielsen J, Ravn H. Sex-differential non-specific effects of BCG and DTP in Cebu, the Philippines. Int. J. Epidemiol. 38(1), 320-323; author reply 323-324 (2009).

16 Aaby P, Roth A, Ravn H et al. Randomized trial of BCG vaccination at birth to low-birth-weight children: beneficial nonspecific effects in the neonatal period? J. Infect. Dis. 204(2), 245-252 (2011).

17 Biering-Sorensen S, Andersen A, Ravn H, Monterio I, Aaby P, Benn CS. Early BCG vaccine to low-birth-weight infants and the effects on growth in the first year of life: a randomised controlled trial. BMC Pediatr. 15, 137 (2015).

18 Jensen KJ, Larsen N, Biering-Sorensen S et al. Heterologous immunological effects of early BCG vaccination in low-birth-weight infants in Guinea-Bissau: a randomized-controlled trial. J. Infect. Dis. 211(6), 956-967 (2015).

19 Roth AE, Stensballe LG, Garly ML, Aaby P. Beneficial non-targeted effects of BCG - ethical implications for the coming introduction of new TB vaccines. Tuberculosis 86(6), 397-403 (2006).

20 Buffen K, Oosting M, Quintin J et al. Autophagy controls BCG-induced trained immunity and the response to intravesical BCG therapy for bladder cancer. PLoS Pathog. 10(10), e1004485 (2014).

21 Kleinnijenhuis J, Quintin J, Preijers F et al. BCG-induced trained immunity in NK cells: role for non-specific protection to infection. Clin. Immunol. 155(2), 213-219 (2014).

22 Kleinnijenhuis J, Van Crevel R, Netea MG. Trained immunity: consequences for the heterologous effects of BCG vaccination. Trans. R. Soc. Trop. Med. Hyg. 109(1), 29-35 (2015).
23 Hoft DF, Worku S, Kampmann B et al. Investigation of the relationships between immune-mediated inhibition of mycobacterial growth and other potential surrogate markers of protective Mycobacterium tuberculosis immunity. J. Infect. Dis. 186(10), 1448-1457 (2002).

24 Kampmann B, Gaora PO, Snewin VA, Gares MP, Young DB, Levin M. Evaluation of human antimycobacterial immunity using recombinant reporter mycobacteria. J. Infect. Dis. 182(3), 895-901 (2000).

25 Silver RF, Li Q, Boom WH, Ellner JJ. Lymphocyte-dependent inhibition of growth of virulent Mycobacterium tuberculosis $\mathrm{H} 37 \mathrm{Rv}$ within human monocytes: requirement for $\mathrm{CD}^{+} \mathrm{T}$ cells in purified protein derivativepositive, but not in purified protein derivative-negative subjects. J. Immunol. 160(5), 2408-2417 (1998).

26 Worku S, Hoft DF. In vitro measurement of protective mycobacterial immunity: antigen-specific expansion of $\mathrm{T}$ cells capable of inhibiting intracellular growth of Bacille Calmette-Guerin. Clin. Infect. Dis. 30(Suppl. 3), S257-S261 (2000)

27 Fletcher HA, Tanner R, Wallis RS et al. Inhibition of mycobacterial growth in vitro following primary but not secondary vaccination with Mycobacterium bovis BCG. Clin. Vacc. Immunol. 20(11), 1683-1689 (2013).

28 Matsumiya M, Satti I, Chomka A et al. Gene expression and cytokine profile correlate with mycobacterial growth in a human BCG challenge model. J. Infect. Dis. 211(9), 1499-1509 (2015).

29 Harris SA, Meyer J, Satti I et al. Evaluation of a human BCG challenge model to assess antimycobacterial immunity induced by BCG and a candidate tuberculosis vaccine, MVA85A, alone and in combination. J. Infect. Dis. 209(8), 1259-1268 (2014).

30 Minassian AM, Satti I, Poulton ID, Meyer J, Hill AV, Mcshane H. A human challenge model for Mycobacterium tuberculosis using Mycobacterium bovis Bacille Calmette-Guerin. J. Infect. Dis. 205(7), 1035-1042 (2012).

31 Chiacchio T, Petruccioli E, Vanini V et al. Polyfunctional T-cells and effector memory phenotype are associated with active $\mathrm{TB}$ in HIV-infected patients. J. Infect. 69(6), 533-545 (2014).

32 Petruccioli E, Petrone L, Vanini V et al. IFNgamma/TNFalpha specific-cells and effector memory phenotype associate with active tuberculosis. J. Infect. 66(6), 475-486 (2013). 
Vot Patrizia A, Vigano S et al. Combined use of Mycobacterium tuberculosis-specific CD4 and CD8 T-cell responses is a powerful diagnostic tool of active tuberculosis. Clin. Infect. Dis. 60(3), 432-437 (2015).

34 Rozot V, Vigano S, Mazza-Stalder J et al. Mycobacterium tuberculosis-specific CD8 ${ }^{+}$ $\mathrm{T}$ cells are functionally and phenotypically different between latent infection and active disease. Eur. J. Immunol. 43(6), 1568-1577 (2013).

35 Streitz M, Fuhrmann S, Powell F et al. Tuberculin-specific $T$ cells are reduced in active pulmonary tuberculosis compared with LTBI or status post BCG vaccination. J. Infect. Dis. 203(3), 378-382 (2011).

36 Harari A, Rozot V, Bellutti Enders F et al. Dominant TNF-alpha ${ }^{+}$Mycobacterium tuberculosis-specific CD4 ${ }^{+} \mathrm{T}$ cell responses discriminate between latent infection and active disease. Nat. Med. 17(3), 372-376 (2011).

37 Berry MP, Graham CM, Mcnab FW et al. An interferon-inducible neutrophil-driven blood transcriptional signature in human tuberculosis. Nature 466(7309), 973-977 (2010).

-. Identification of myeloid cells and IFN-1 as a potential immune mechanism of importance for disease.

38 Cliff JM, Lee JS, Constantinou $\mathrm{N}$ et al. Distinct phases of blood gene expression pattern through tuberculosis treatment reflect modulation of the humoral immune response. J. Infect. Dis. 207(1), 18-29 (2013).

39 Maertzdorf J, Repsilber D, Parida SK et al. Human gene expression profiles of susceptibility and resistance in tuberculosis. Genes Immun. 12(1), 15-22 (2011).
40 Anderson ST, Kaforou M, Brent AJ et al. Diagnosis of childhood tuberculosis and host RNA expression in Africa. N. Engl. J. Med. 370(18), 1712-1723 (2014).

41 Kaforou M, Wright VJ, Oni T et al. Detection of tuberculosis in HIV-infected and -uninfected African adults using whole blood RNA expression signatures: a case-control study. PLoS Med. 10(10), e1001538 (2013).

42 Zak DE, Penn-Nicholson A, Scriba TJ et al. A blood RNA signature for tuberculosis diseaserisk: a prospective cohort study. Lancet pii: S0140-6736(15)01316-1, doi:10.1016/ S0140-6736(15)01316-1 (2016) (Epub ahead of print).

43 Bloom CI, Graham CM, Berry MP et al. Transcriptional blood signatures distinguish pulmonary tuberculosis, pulmonary sarcoidosis, pneumonias and lung cancers. PLoS ONE 8(8), e70630 (2013).

44 van Crevel R, Dockrell HM TANDEM Consortium. TANDEM: understanding diabetes and tuberculosis. Lancet Diabetes Endocrinol. 2(4), 270-272 (2014).

45 Altare F, Durandy A, Lammas D et al. Impairment of mycobacterial immunity in human interleukin-12 receptor deficiency. Science 280(5368), 1432-1435 (1998).

46 Cooper AM, Dalton DK, Stewart TA, Griffin JP, Russell DG, Orme IM. Disseminated tuberculosis in interferon gamma genedisrupted mice. J. Exp. Med. 178(6), 2243-2247 (1993).

47 Flynn JL, Chan J, Triebold KJ, Dalton DK, Stewart TA, Bloom BR. An essential role for interferon gamma in resistance to Mycobacterium tuberculosis infection. J. Exp. Med. 178(6), 2249-2254 (1993).
48 Jouanguy E, Altare F, Lamhamedi S et al. Interferon-gamma-receptor deficiency in an infant with fatal Bacille Calmette-Guerin infection. N. Engl. J. Med. 335(26), 1956-1961 (1996).

49 Lichtenauer-Kaligis EG, De Boer T, Verreck FA et al. Severe Mycobacterium bovis BCG infections in a large series of novel IL-12 receptor betal deficient patients and evidence for the existence of partial IL-12 receptor beta1 deficiency. Eur. J. Immunol. 33(1), 59-69 (2003)

50 Newport MJ, Huxley CM, Huston S et al. A mutation in the interferon-gamma-receptor gene and susceptibility to mycobacterial infection. N. Engl. J. Med. 335(26), 1941-1949 (1996).

51 Picard C, Fieschi C, Altare F et al. Inherited interleukin-12 deficiency: IL12B genotype and clinical phenotype of 13 patients from six kindreds. Am. J. Hum. Genet. 70(2), 336-348 (2002).

52 Kagina BM, Abel B, Scriba TJ et al. Specific $\mathrm{T}$ cell frequency and cytokine expression profile do not correlate with protection against tuberculosis after Bacillus Calmette-Guerin vaccination of newborns. Am. J. Respir. Crit. Care Med. 182(8), 1073-1079 (2010).

53 Ndiaye BP, Thienemann F, Ota M et al. Safety, immunogenicity, and efficacy of the candidate tuberculosis vaccine MVA85A in healthy adults infected with HIV-1: a randomised, placebo-controlled, Phase 2 trial. Lancet Respir. Med. 3(3), 190-200 (2015).

54 TuBerculosis Vaccine Initiative. www.tbvi.eu 